\title{
Criminologie
}

\section{Gibier de recherche, la police et le projet de connaître}

\section{Dominique Monjardet}

Volume 38, numéro 2, automne 2005

La police en pièces détachées

URI : https://id.erudit.org/iderudit/012660ar

DOI : https://doi.org/10.7202/012660ar

Aller au sommaire du numéro

Éditeur(s)

Les Presses de l'Université de Montréal

ISSN

0316-0041 (imprimé)

1492-1367 (numérique)

Découvrir la revue

Citer cet article

Monjardet, D. (2005). Gibier de recherche, la police et le projet de connaître. Criminologie, 38(2), 13-37. https://doi.org/10.7202/012660ar

\section{Résumé de l'article}

On explore les rapports entre les policiers, l'institution policière et la recherche en sciences sociales sur la police. À cette fin, l'expérience française des recherches sur la police depuis le début des années 80 est retracée. Les différentes formes de résistance à la recherche sont identifiées et détaillées. On tente de les interpréter en mettant en évidence, au-delà de l'incompréhension de la spécificité de la démarche du chercheur (par rapport à celle de l'enquêteur policier), la portée et les enjeux de l'objectivation des savoirs policiers au regard de deux propriétés majeures de l'organisation policière. Le caractère collectif du travail au niveau des exécutants, d'une part, et, d'autre part, les relations incertaines entre compétence professionnelle et position hiérarchique dans une organisation bureaucratique. Il est possible que la nouvelle insistance du politique sur les « résultats » attendus de l'action policière modifie sensiblement les termes de cette relation, de telle sorte que les résultats de la recherche puissent apparaître comme une ressource pour les éléments professionnalisés du corps policier.
Ce document est protégé par la loi sur le droit d'auteur. L'utilisation des services d’Érudit (y compris la reproduction) est assujettie à sa politique d'utilisation que vous pouvez consulter en ligne.

https://apropos.erudit.org/fr/usagers/politique-dutilisation/ 


\title{
Gibier de recherche, la police et le projet de connaître ${ }^{1}$
}

\author{
Dominique Monjardet \\ CERSA (Centre d'Étude et de recherche en science administrative) \\ Centre national de la Recherche scientifique (CNRS) \\ Université Paris \\ dominique.monjardet@u-paris2.fr
}

RÉSUMÉ - On explore les rapports entre les policiers, l'institution policière et la recherche en sciences sociales sur la police. À cette fin, l'expérience française des recherches sur la police depuis le début des années 80 est retracée. Les différentes formes de résistance à la recherche sont identifiées et détaillées. On tente de les interpréter en mettant en évidence, au-delà de l'incompréhension de la spécificité de la démarche du chercheur (par rapport à celle de l'enquêteur policier), la portée et les enjeux de l'objectivation des savoirs policiers au regard de deux propriétés majeures de l'organisation policière. Le caractère collectif du travail au niveau des exécutants, $d^{\prime}$ 'une part, et, d'autre part, les relations incertaines entre compétence professionnelle et position hiérarchique dans une organisation bureaucratique. Il est possible que la nouvelle insistance du politique sur les «résultats» attendus de l'action policière modifie sensiblement les termes de cette relation, de telle sorte que les résultats de la recherche puissent apparaître comme une ressource pour les éléments professionnalisés du corps policier.

ABSTRACT - This article explores the relationships between the police, the police institution and social research on policing. Its prime object is the French experience with police studies since the early 1980s, which is first described. Various forms of obstacles to research are identified and examined in detail. Notwithstanding the misunderstandings surrounding the specific character of a researcher's approach as compared to a police investigator's, the preceding obstacles are interpreted by focussing on the new dynamics between the production of objective knowledge on the police and between two major features of police organisations. These two features are on the one hand the collective nature of the work that is performed at the level of the police rank and file and, on the other hand, the problematic nature of the relationship between one's competence and one's ranking within the pyramidal structure of bureaucracies. It is

1. Merci à Antoinette Chauvenet et à Frédéric Ocqueteau, qui ont fait d'une première version de ce texte une lecture acérée. 
hypothesised that the new emphasis of government on getting ever increasing results out of policing will change the dynamics between research and the police institution, research being viewed as a new instrumental resource for the ranking officers of the organisation.

Dans l'article séminal qui ouvrait, il y a 20 ans, la livraison que cette même revue consacrait à la police, Jean-Paul Brodeur notait que «l'action policière est un objet qui oppose une résistance délibérée au projet de connaître (version révisée dans Brodeur (2003)) ». L'expérience française des recherches en sciences sociales sur les polices depuis un quart de siècle ne dément pas ce jugement. Elle témoigne que la recherche externe sur la police a été, est et restera vraisemblablement difficile, sous le triple rapport de l'accès au terrain, de la collecte des données et de la réception des résultats. On présentera un relevé rapide de ces difficultés avant de tenter d'en comprendre les raisons.

\section{La résistance à la recherche}

1. Sauf en de rarissimes et très discrètes exceptions, toute sollicitation d'ouverture de terrain auprès d'un service de police quelconque fait l'objet, soit d'un refus immédiat soit du renvoi auprès de l'autorité supérieure, jusqu'à la direction centrale au ministère de l'Intérieur à Paris. Jusqu'en 1981, cette dernière oppose un refus systématique auquel on ne connaît qu'une seule et très mince exception: A. Davidovitch avait eu accès aux mains courantes de deux commissariats. À partir de 1981 se produit une ouverture limitée sous l'impulsion de la nouvelle direction de la formation de la police². En 1989, la création de l'Institut des hautes études de sécurité intérieure (IHÉSI) et, en son sein, d'un département de recherche permet à la communauté scientifique de disposer d'un accès plus ouvert aux terrains policiers, c'est toutefois toujours l'administration qui détient, en dernier ressort, les autorisations d'accès, avec deux conséquences notables. Des pans entiers de l'activité policière sont restés à l'abri de toute recherche (le renseignement, par exemple), et la propension à l'ouverture de terrains est très sensible à la conjoncture politique (Monjardet, 1997; Ocqueteau et Monjardet,

2. La démarche est retracée par son responsable, Jean-Marc Erbès, dans sa postface à D. Monjardet (1996). Voir aussi le point de vue de Loubet del Bayle (1999). 
2005) : les variations de celle-ci placent alternativement en position décisionnelle les fractions les plus ouvertes et les fractions les plus fermées de la haute hiérarchie policière et préfectorale $e^{3}$ le volume des opérations de recherche autorisées connaît ainsi des fluctuations considérables.

2. Contrepartie de cette centralisation de la décision d'accès: lorsque celle-ci est accordée par l'autorité supérieure, le service localement concerné ne saurait y faire opposition. Le chercheur voit ainsi garantie la possibilité de sa présence dans les locaux policiers désignés. Pour autant, on s'en doute, cette seule présence, imposée par une autorité supérieure, ne garantit nullement l'accès aux informations recherchées, qui dépend pour l'essentiel de la bonne volonté dont vont faire preuve, ou non, les personnels. Cette situation est commune à toute investigation dans une organisation de travail hiérarchisée, et, de ce point de vue, se retrouvent dans un commissariat de sécurité publique, une direction de police judiciaire ou une compagnie républicaine de sécurité, les mêmes difficultés, et facilités, que l'on peut retrouver dans une usine, une entreprise commerciale, un hôpital ou une administration. Pour avoir pu comparer pendant quelques lustres les situations d'enquête en milieu industriel et en milieu policier, on a cru pouvoir noter cependant que certaines résistances communes présentaient dans ce dernier cas des formes plus prononcées. On peut désigner sous ce chapitre le recours plus fréquent qu'ailleurs à la langue de bois, dont la forme spécifiquement policière est le recours systématique au seul énoncé de la règle (loi, règlement, procédure, consigne) supposée guider toute conduite. Significativement, la langue de bois se fait d'autant plus fréquente et épaisse qu'on s'élève dans la hiérarchie ${ }^{4}$, et il est infiniment plus difficile de mener un entretien d'enquête avec un commissaire qu'avec un gardien, a fortiori s'agissant de l'observation directe du travail de l'un et de l'autre.

L'existence d'un «secret professionnel» permet également, à qui veut en faire usage, de donner une extension considérable à ce qui échappe ainsi à l'investigation. Toute série d'entretiens démontre à l'envi que ce recours est discrétionnaire: telle «affaire» que l'un aura couverte du voile du secret sera minutieusement décrite par l'autre...

3. Traditionnellement, les postes de direction de la police nationale au ministère de l'Intérieur sont partagés entre les sommets de la profession policière (contrôleurs généraux, inspecteurs généraux) et le corps préfectoral. 
Plus spécifique encore, et obstacle plus redoutable, est la réciprocité de positions que la situation d'entretien fait parfois émerger. Il arrive que le policier interrogé par le chercheur se perçoive tout à coup comme objet d'une «enquête», c'est-à-dire dans la position du "mis en cause», alors que c'est d'ordinaire lui qui pose les questions... Lorsqu'elle se produit, cette perception peut être brutale et mettre instantanément fin à la relation. Il fait aussi partie de la compétence du chercheur de minimiser ces occurrences. Par exemple, en refusant l'offre souvent faite de s'installer "pour être plus à l'aise pour prendre vos notes» au bureau de l'intéressé : c'est alors - ayant pris sa place - que le risque d'inversion de position est plus grand. Sur ces questions, consulter Chauvenet et Orlic (1985).

Il est toutefois d'expérience commune aux chercheurs ayant pratiqué les terrains policiers que la difficulté majeure est dans l'accès. Une fois celui-ci obtenu, l'accueil du plus grand nombre est très ouvert, et le chercheur est plus souvent submergé d'informations (pas toujours sollicitées) que tenu à l'écart. On ne connaît pas dans le cas français de situations comparables à celles qu'ont vécu, au début des années 50 , W. Westley, à Gary (Ind., É.-U.), boycotté par l'ensemble des personnels pendant plusieurs semaines (Westley, 1970), ou, dans les années 60, M. Punch à Amsterdam, que des menaces physiques ont amené à renoncer à son enquête (Punch, 1986).

C'est au troisième stade de l'enquête sociologique, celui de la restitution des résultats, que la résistance policière apparât la plus vive.

3. La première forme de cette résistance est l'ignorance, ou refus de savoir. Les recherches menées sous l'égide de l'IHÉSI font d'ordinaire l'objet de deux types de restitutions. Restitutions orales, sous forme de séances ad hoc, dans les locaux de l'Institut, auxquels sont conviés, très largement, policiers qui ont été l'objet de l'enquête, responsables des services et de la direction concernés, policiers affectés aux autres départements de l'Institut (formation et études notamment), etc. La présence des uns comme des autres est ordinairement infinitésimale. Quant à la

4. H. Souchon, alors commissaire de police et professeur à l'école des commissaires (ENSP), a mené, au début des années 80 , une enquête sur les pratiques d'admonestation par questionnaire écrit auprès de ses collègues. Il signale dans son rapport (De l'exercice du pouvoir discrétionnaire par les organes de police, multigraphié, 1981), avoir reçu nombre de refus motivés par le fait que l'admonestation n'étant pas prévue par le code pénal, il est hors de question d'en décrire la pratique. 
restitution écrite, sous forme de rapport, article, ouvrage, il est d'observation constante que rarissimes sont les policiers qui en prennent connaissance, ne fut-ce de la façon la plus superficielle ${ }^{5}$. Lorsque cependant ces résultats parviennent à la connaissance des policiers, sous une forme ou sous une autre, s'expriment d'autres formes de résistance, les diverses techniques de disqualification. On en recense quatre principales:

3.1. En premier lieu, l'erreur manifeste. Il arrive que sur tel ou tel point de technique professionnelle, l'observateur ait fait une erreur: il s'est perdu dans les détails de la procédure pénale à l'occasion du récit de telle affaire, ou a confondu deux règles différentes de calcul des heures supplémentaires pour les unités en déplacement hors de leur circonscription. D'ordinaire l'erreur est minime, et n'entache pas gravement la compréhension de la situation, relation ou action décrite. Elle permettra néanmoins de témoigner que «le chercheur ne connaît rien à ce dont il parle» et par là de disqualifier l'ensemble de son propos.

3.2. En second lieu, la pertinence des données recueillies est niée. Soit l'exemple de la recherche "cohorte» menée en 1992-1993 auprès d'une promotion entière d'élèves gardiens de la paix. Ceux-ci ont répondu au même questionnaire lors de leur entrée en école de police en janvier 1992, puis lors de la dernière semaine de scolarité en janvier $1993^{6}$. L'analyse des questionnaires témoigne, entre autres choses, des effets de la formation transmise. Le tri des résultats selon les sept écoles concernées témoigne surabondamment que la performance pédagogique de celles-ci est très inégale. Mis en présence de ces résultats, le directeur de la formation de la police nationale tranchera: "On sait bien qu'à ce genre de questionnaire les gens répondent n'importe quoi». L'enquête sera poursuivie auprès de la même population jusqu'en 2002 (interrogations en 1994, 1997, 2002) et les services de la

5. On consultera, par exemple, la bibliographie utilisée par Rudolph (2001), contrôleur général à l'Inspection générale de la police nationale, pour nourrir sa réflexion sur «le policier et le chercheur». Sur les 23 ouvrages cités, deux prennent en compte des recherches engagées sous l'égide de l'IHÉSI.

6 . Au moment où cette recherche commence, en 1992, la direction de la formation de la PN dispose de 13 écoles, réparties sur tout le territoire. La $121^{\mathrm{e}}$ promotion des élèves gardiens de la paix, objet de notre recherche, est ainsi répartie entre sept de ces écoles, qui vont scolariser chacune pendant une année de 90 à 215 recrues, pour un total de 1166. Les rapports correspondant aux six phases de cette recherche sont déposés à l'INHES où ils sont consultables (Monjardet et Gorgeon, 1993). Une publication d'ensemble est en préparation. 
formation ne manifesteront d'autre intérêt à son égard que la tentative de l'interdire (en 1997, le commissaire adjoint auprès du directeur de la formation signe une note déconseillant la poursuite de cette recherche). De la même façon, les données de l'enquête Interface, menée par la nouvelle direction de la formation en 1981-82 auprès des 110000 policiers alors en fonction, qui avait reçu plus de 70000 réponses, et constituait donc la base de données la plus ample jamais constituée sur la police française, ont fait l'objet d'une tentative de discrédit par le biais d'une rumeur selon laquelle, les questionnaires étant anonymes, les gardiens de la paix et autres inspecteurs avaient massivement usurpé une qualité de commissaire. Bien évidemment tous les contrôles internes de cohérence, dont est doté tout questionnaire bien conçu, infirmaient cette hypothèse.

3.3. Troisième forme de disqualification, lorsque les données ne sont ni erronées ni impertinentes, elles sont réputées «exceptionnelles», reflétant des situations tout à fait particulières ne pouvant soutenir la moindre généralisation. Cet argument a deux faces. En première analyse, il s'entend comme localisme: le hasard aurait voulu que le chercheur observe une situation rare ou rarissime, par exemple, le vestige d'anciennes coutumes qui sont réputées disparues partout ailleurs, ou une situation explicitement déviante, et donc vouée à être redressée au plus tôt. Dans les deux cas, les conclusions qu'il tire de ces observations sont nécessairement infondées. On verra par la suite que l'exception est aussi invoquée comme caractéristique substantielle de la tâche policière: celle-ci n'est pas objectivable par les procédés habituels du chercheur parce qu'elle est surdéterminée par «l'événement», occurrence singulière par définition.

3.4. Lorsque enfin, il n'est possible d'opposer au chercheur aucune contestation de son matériel, la dernière ligne de défense est, à l'exact opposé des précédentes, le constat de trivialité: «On savait cela depuis toujours, la recherche ne nous apprend rien». L'argument a, plus que les précédents, une apparence de vraisemblance. Sachant que les données que produit le chercheur lui ont été fournies pour l'essentiel par les policiers eux-mêmes, il n'est pas surprenant que ceux-ci y retrouvent ce qu'ils y ont mis. L'argument méconnaît toutefois deux apports de la recherche. Le premier est la confrontation des savoirs policiers, qui sont pluralistes, et dans nombre de domaines hétérogènes, voire contradictoires. Leur confrontation est, pour une part, l'objet même, en même 
temps qu'une ressource importante de la recherche. Mais plus encore, la valeur ajoutée de la recherche est dans l'énoncé de ces savoirs jusqu'alors implicites, dans la publicité qui est donnée à ce qui, peut-être, était su de tous, mais restait informulé. Se loge ici un des malentendus majeurs entre policiers et chercheurs, et qui différencie radicalement l'enquête scientifique de l'enquête policière. Il ne s'agit pas pour le chercheur de percer des secrets, de dévoiler ce qu'on entend lui dissimuler, de révéler «des choses cachées depuis le commencement du monde», mais de comprendre, c'est-à-dire de mettre en relation.

L'exemple des «cadavres dans les placards » illustre cette distinction essentielle. Dans deux services où j'ai pu effectuer une enquête de longue durée (plusieurs mois de présence intensive), les supposés cadavres dans les placards ont fait l'objet d'un traitement distinct. Dans le premier, le chef de service m'a informé en m'accueillant que j'avais pleine liberté de mener mon enquête comme je voulais, et que je ne trouverais pas de «cadavres dans le placard», parce qu'il n'y en avait pas. Le second a réuni ses cadres avant mon arrivée, et a dressé avec eux la liste de ce qu'il était souhaitable de me laisser ignorer. Dans le premier cas, j'ai trouvé chemin faisant quelques placards moins nets que ce qu'on m'en avait dit, et j'ai tout lieu de supposer que le chef de service les ignorait lui-même. Dans le second, il s'est évidemment trouvé un «traître» pour m'informer par le menu de ce que je n'étais pas censé savoir, et d'abord de la réunion qui en avait dressé l'inventaire. On ne trouvera aucune mention de ces «cadavres» dans le rapport de recherche correspondant. Non pas que le chercheur ne se soit autocensuré, mais parce que ces informations n'étaient pas utiles au regard de l'objet de recherche. Dans les deux cas, il s'agissait de cas patents de déviances policières (violences dans le premier, corruption dans le second) qui n'apportaient pas d'élément de connaissance supplémentaire par rapport à ce que j'avais rassemblé, par ailleurs, quant au fonctionnement de la chaîne hiérarchique (prescription, contrôle). Ces anecdotes emportent cependant un savoir, mais qui n'est pas celui que les policiers supposent : elles pointent la difficulté qu'ont les policiers à différencier opérations de recherche scientifique, curiosité journalistique et procédures de contrôle. Saturés par ces dernières, excédés par la presse, ils y assimilent les premières, et y opposent une résistance de même nature. Les rapports de la police et de la presse sont singuliers. On sait que la première instrumente très largement celle-ci, en laissant filtrer l'information tout autant qu'en la retenant. Par ailleurs, toute étude de presse montre que le sort qui y est 
fait à l'action policière est très majoritairement positif. Pour autant, la corporation policière est convaincue d'être maltraitée par les médias. C'est le premier point sur lequel les recrues en école de police vont s'accorder : ils sont $74 \%$ en entrant à l'école pour juger que «les médias donnent une image de la police: plutôt défavorable», cette proportion monte à $93 \%$ à la sortie d'école et après dix ans d'ancienneté, elle s'établit à $88 \%$ (recherche «cohorte», op. cit.). (Voir le numéro spécial «Police et médias» des Cahiers de la sécurité intérieure nº 12, 1993.)

Sous ces différentes modalités s'exprime ainsi la « résistance au projet de connaître» des policiers, qui est à la fois par escalade successive du plus spontané au plus raffiné:

- résistance à l'intrusion d'un regard extérieur,

- revendication d'un monopole de compétence et déni de la pertinence d'un regard profane,

- affirmation de la singularité irréductible des choses policières, auxquelles ne sauraient donc s'appliquer des savoirs nés dans d'autres champs,

- refus de la sélection et de l'objectivation des savoir-faire efficaces.

\section{Les savoirs policiers}

On a déjà largement documenté les deux premiers points, qui s'illustrent, entre autres, par les différentes formes de résistance à la recherche externe. C'est ici le lieu de noter qu'ils ne sont pas propres à la profession policière. La plupart des professions fermées, c'est-à-dire celles auxquelles on n'accède que par une procédure d'habilitation contrôlée par la profession elle-même (l'exemple type est celui de la médecine) nourrissent aussi une grande défiance à l'égard de toute investigation externe et opposent le monopole de savoirs détenus par le clerc à l'incompétence radicale du laïc. La récente recherche de Latour (2002) sur le Conseil d'État a reçu le même accueil de la part de nombre de juristes: dès qu'est repérée dans l'analyse du sociologue une imprécision ou erreur sur le point de droit le plus mince, son incompétence est emphatiquement soulignée, et l'ensemble de son travail disqualifié. On trouverait sans peine des exemples similaires dans nombre de professions.

Par contre, les deux points suivants sont plus singuliers et définissent un rapport au savoir professionnel assez particulier. On notera ici, à l'articulation entre la revendication du monopole de savoir et la nature même de ce savoir une particularité des polices françaises, qui illustre bien une position originale (ou extrême) sur ce sujet. Dans l'ensemble 
de la police nationale, qui comptait, en 20037, 133023 agents, on recense 15301 personnels administratifs, ouvriers et de service, soit $11 \%$. Ou inversement: neuf emplois sur dix sont tenus par des policiers. Le Service de police de la ville de Montréal emploie 994 civils pour 4172 policiers, soit $18,3 \%$ (budget 2004). On estime que, dans les polices britanniques, les emplois tenus par des non-policiers constituent le tiers de l'effectif global (Reiner, 1993). Ce rapport du simple au double ou au triple indique bien que la conception française du monopole policier est très extensive. Elle se traduit, par exemple, par le fait que dans les directions centrales de la police nationale, les responsables des bureaux du personnel, du matériel, du budget, de la statistique ou de l'informatique sont des commissaires de police et non des spécialistes du domaine. Il n'est pas assuré que ces responsables, par définition autodidactes dans leur champ de responsabilité administrative, en détiennent toujours les compétences propres, mais leur position même signifie qu'au regard de l'administration de la police, la qualification policière prime toutes les autres.

Troisième type de recours contre les savoirs externes, l'invocation de la singularité supposée de la tâche policière est à vrai dire un argument à éclipser. Lorsqu'il est question des fautes professionnelles commises par des policiers (ou «bavures»), le chercheur est au contraire systématiquement prié de les «situer dans une approche comparative concernant l'ensemble des professions (on pense en particulier aux professions médicales ou journalistiques)» (Rudolph, 2001: 98) ${ }^{8}$. Hormis ce cas, non négligeable, elle fonde cependant un isolationnisme cognitif radical: il n'y a ni emprunt ni transfert de connaissances, notamment dans les structures de la formation initiale, entre la police et les autres groupes professionnels en charge, par exemple, de situations d'urgence et crises (corps de pompiers, urgences médicales), ou des situations de conflit (travail social, médiation, armées). Dans le champ policier lui-même, il n'a jamais semblé pertinent de transférer les savoirs reconnus efficaces dans le domaine de la police de la route (prévention des accidents) à la

7. Rapport annuel, p. 98. (Hors adjoints de sécurité, employés sur un contrat temporaire.)

8. On ajoute d'ordinaire que la proportion des policiers corrompus ou violents n'est sans doute pas supérieure à celle des instituteurs pédophiles, des notaires indélicats et des chirurgiens maladroits. Le raisonnement selon lequel l'existence de ceux-ci relativiserait la faute de ceux-là apparaît d'autant plus obscur que les médecins ou les notaires ne sont pas spécifiquement chargés de faire appliquer la loi par l'ensemble des citoyens. 
prévention d'autres formes de délinquance. A fortiori, les savoirs constitués dans le domaine du fonctionnement des grandes organisations hiérarchisées (organisations industrielles, administratives, de service) sont radicalement ignorés. Qu'il s'agisse de motivation professionnelle ou de satisfaction au travail, de contrôle ou de mesure de l'activité, de normes collectives informelles ou de gestion du changement, tous domaines dans lesquels sociologie, psychologie sociale, sciences de la gestion et du management ont accumulé des savoirs solides, le corps policier et son administration ne se fient qu'à l'expertise «indigène», d'autant plus indigente qu'elle récuse par principe toute confrontation et la nécessité de tout apprentissage ${ }^{9}$. Il faut ajouter ici que les sciences sociales elles-mêmes ont parfois apporté de l'eau à ce moulin. L'analyse de Skolnick (1966) selon qui la situation de travail des policiers serait unique, car ils sont seuls à combiner l'exposition au danger (comme les militaires), une relation d'autorité avec le public (comme les enseignants) et le souci de l'efficacité de leur travail (comme les travailleurs de l'industrie) est célèbre. Skolnick en déduisait la détention de «lunettes cognitives» particulières, au principe d'une «working personality» propre, nourrissant une culture professionnelle homogène. Toutefois, toutes les recherches empiriques menées depuis lors témoignent au contraire que, hormis un petit noyau de stéréotypes partagés, le monde policier est infiniment plus divers que ne le supposait Skolnick. Il faut en retour en inférer que les «situations de travail» policières ne sont peut-être pas aussi radicalement particulières que ce dernier ne le pensait, et que les policiers ne l'affirment.

Enfin, la résistance policière à la recherche soutient et exprime un refus de l'objectivation des savoir-faire efficaces. Sur ce dernier point, il est frappant de constater que les policiers ont réussi à conserver l'exclusivité de la formation professionnelle de leurs recrues, tout en évitant de concevoir les instruments de cette formation, de formaliser les savoirs transmis (hormis le champ très précisément délimité des GTPI (gestes techniques et professionnels d'intervention), et de la police dite «tech-

9. La mise en œuvre de la réforme de "police de proximité» en 1999-2001 a constitué un exemple achevé des conséquences d'une telle posture. Attelée à une réforme de très grande ampleur portant à la fois sur l'organisation et l'orientation du travail de sécurité publique, l'administration a procédé à ce qu'elle sait faire, le tracé et l'imposition de nouveaux organigrammes, et, pour le reste, recouru à des pratiques magiques comme l'invocation d'une nécessaire «révolution culturelle» (Sur ce cas, voir D. Monjardet, 2002). 
nique et scientifique», qui recourt à l'expertise scientifique $)^{10}$. Ce refus peut paraître comme conséquence des trois traits précédents, et du même mouvement comme une carence : qu'est-ce donc que cette "profession» qui ne revendique pas un corps de savoir identifiable, objectivé, transmissible? Où se loge le «professionnalisme» d'un métier dépourvu de tout «traité » ou «manuel»? Il y a une interprétation plus convaincante, qui repose sur deux ordres de raisons, toutes deux relevant de cette sociologie du travail policier que la police s'emploie à méconnaitre.

En premier lieu, le refus de l'objectivation traduit, indirectement, deux traits majeurs du travail policier:

- celui-ci est le plus souvent collectif. Dans le quotidien de la police urbaine, l'unité de travail est la patrouille, la brigade, le groupe d'enquête, plus que l'individu;

- dans le travail collectif, sont indissociables les compétences individuelles mobilisées (qualifications, savoir-faire) et les normes collectives de leur mise en œuvre. Dans la très grande majorité des cas, ces normes collectives qui règlent l'intensité, la répartition et le contrôle du travail sont informelles, et soutiennent l'autonomie du groupe de travail par rapport à toute tentative de prescription hiérarchique de la tâche et de son mode opératoire. La nature implicite, défensive, protectrice de ces normes exclut par définition qu'elles soient explicitées par l'organisation (elles sont pour l'essentiel développées et mises en œuvre contre l'organisation formelle du travail, de la hiérarchie et du contrôle).

En conséquence, la compétence, comprise comme combinaison indissoluble de propriétés individuelles et d'adhésion aux normes collectives, ne peut être, dans une bonne part des tâches policières, constituée, identifiée et réalisée hors du collectif de travail lui-même. Ce qui fonde le précepte universel ${ }^{11}$ à l'égard des nouveaux affectés sortant d'école: "Oublie tout ce qu'on t'a mis dans le crâne à l'école, tu ne sais rien, c'est ici et maintenant que commence ton apprentissage ». Et le propos est littéralement exact: il est hors de question qu'un nouveau venu excipe d'un savoir extérieur à ce que le collectif de travail qui l'accueille reconnaît et sanctionne. En tout état de cause, ce savoir serait,

10. L' IHÉSI a également joué un rôle pionnier à cet égard, en suscitant la recherche de S. Tiévant (2001: 98). Les savoir-faire en police de proximité. À ce jour, (2005), cette initiative n'a pas fait école.

11. On le retrouve à l'identique aussi bien dans nos observations personnelles en France que dans celles de J. Van Maanen (1975) aux États-Unis ou de N. Fielding (1988) en Angleterre. 
par construction, impertinent puisqu'il n'incorpore pas ses modalités sociales de mise en œuvre. Le précepte ainsi ne doit pas se comprendre comme «On t'a mis dans le crâne à l'école un savoir faux, erroné» (ce qui est d'autant plus difficile à soutenir que les enseignants sont tous des collègues), mais sous la forme «ce qu'on t'a enseigné à l'école est purement théorique, cela ne s'actualise et ne prend sens que par et dans la pratique du collectif dans lequel tu entres, et c'est ce collectif qui est seul habilité à juger de la pertinence de tes savoirs ». C'est par la médiation de ce collectif qu'un savoir policier pertinent peut se revendiquer. On dira si on veut, d'une autre façon, que la compétence professionnelle policière est avant tout et entièrement «culturelle»: système de valeurs autant que répertoire de pratiques.

Cette interprétation est vérifiée par les exceptions à la règle, qui sont de deux ordres:

- quand l'organisation elle-même sanctionne le caractère collectif du savoir professionnel. Le cas exemplaire est celui des unités spécialisées de maintien de l'ordre: on sait que la «compétence» d'une CRS ou d'un EGM ${ }^{12}$ par exemple est toute entière exprimée par la capacité à agir comme collectif, unité constituée au sein de laquelle s'abolissent, en se totalisant, les prestations individuelles. En ce cas, il est en effet reconnu que le savoir-faire opérationnel est concentré dans le commandement de l'unité, et que la compétence individuelle des membres de l'unité réside dans leur soumission absolue au collectif. De ce fait, un réseau de normes informelles ne fait pas obstacle à l'objectivation de cette compétence, et elle est efficacement transmise aux nouveaux entrants ${ }^{13}$.

- quand le collectif de travail est construit délibérément comme combinaison de savoir-faire individuels. C'est le cas de certaines unités d'élite, comme les GIPN (groupe d'intervention de la police nationale) qui regroupent des savoirs très spécialisés (tir, pénétration, liaison, etc.) pour l'accomplissement de tâches ponctuelles précisément délimitées (situations de crise). Là encore la pratique du débriefing témoigne de ce que l'efficacité est fonction de la capacité à organiser l'action collective, mais celle-ci étant toute entière finalisée, l'écart entre organisation formelle et informelle est minimal, et le savoir-faire peut être objectivé.

12. Escadrons de gendarmerie mobile, unités spécialisées dans le maintien de l'ordre au sein de la gendarmerie nationale, comme les compagnies républicaines de sécurité au sein de la police nationale.

13. Cette description sommaire de la compétence en maintien de l'ordre a été étayée dans notre article (1988): «Le maintien de l'ordre, technique et idéologie professionnelle des C.R.S.». 
Ces deux cas, très différents en apparence, ont quelques points communs qui les différencient radicalement du travail en «service général». Qu'il s'agisse de maintien de l'ordre ou de règlement de crise, on a affaire à des opérations «discrètes", discontinues, finies, où la mission est précisément instituée et définissable ex ante, et exactement évaluable ex post. On dispose dans ce cas des préalables élémentaires de la prescription et du contrôle d'une "production» policière, et donc de l'élaboration progressive de techniques, outils, modes opératoires objectivés. Moins ces conditions sont réunies, plus l'activité est enserrée dans un réseau de normes collectives informelles, moins elle en est détachable, et donc plus ces dernières prennent le premier pas. À la limite, la qualification première du policier devient «être un bon collègue », et c'est cette qualité relationnelle, sociale, qui va trier les savoirs qu'il détient individuellement. Un savoir transmis par l'école, mais qui contredit les routines du groupe va ainsi être immédiatement disqualifié, et inversement, le collègue qui ne sait rien faire sera néanmoins qualifié s'il se comporte en bon élément dans le groupe. Sous ce rapport, la prétention d'un observateur extérieur à analyser les savoirs policiers, à en décomposer, décrire et mettre en relations les éléments constitutifs est assimilée par les policiers à celle de l'école de les transmettre, et se heurte au même scepticisme.

Cette première analyse de la résistance à l'objectivation s'observe en effet sur le terrain, où elle se formalise dans la conception de la qualification policière comme combinaison de qualités individuelles innées et de l'apprentissage «sur le tas»; l'ancienneté dans le service, qui permet d'accumuler l'expérience, en serait du reste le meilleur indicateur (Monjardet, 1988: 115-123). C'est aussi dire qu'elle est d'abord le fait des exécutants, gardiens et premiers niveaux d'encadrement. On a signalé que ce n'est pas au sein de ces groupes que s'exprime le plus violemment l'hostilité à la recherche externe. Pour comprendre celle-ci lorsqu'elle émane des échelons plus élevés de la hiérarchie, et notamment chez les commissaires de police ${ }^{14}$, dont le bagage universitaire ${ }^{15}$

14. Au questionnaire Interface 1981, évoqué supra, le taux de réponse des commissaires est significativement plus faible que celui des autres policiers ( $52 \%$ contre $62 \%$ ), et ils sont également les plus sceptiques quant à l'utilité de l'enquête: $41 \%$ pensent qu'elle "peut être utile» contre $51 \%$ des gardiens de la paix et $58 \%$ des officiers (ministère de l'Intérieur, 1982)

15. On rappelle que dans la police nationale en France, le recrutement se fait directement dans chacun des trois corps (application, encadrement, direction) qui la composent. 
laisserait supposer au contraire moins de défiance envers les sciences sociales, il faut faire intervenir un autre ordre de considération. Au rebours de l'isolationnisme cognitif du monde policier, on le trouvera à nouveau dans la sociologie anglo-saxonne des organisations, et, plus précisément, dans l'antinomie entre professionnalisme et organisation hiérarchique, tel que l'a exploré notamment W. Kornhauser (1962), et à sa suite un ample courant d'études sur les professionnels dans les bureaucraties $^{16}$.

\section{Professionnalisme et bureaucratie}

L'organisation pyramidale de la police nationale en France, qui combine hiérarchie des corps et hiérarchie des structures, est un modèle d'organisation hiérarchique: entre le gardien de la paix en service dans une ville moyenne et le directeur général à Paris s'échelonnent brigadier, officier commandant le corps urbain, commissaire chef de la sécurité publique, commissaire chef de circonscription, directeur départemental, directeur central. Vont s'intercaler de surcroît des autorités latérales, fonctionnelles, hiérarchiques ou les deux à la fois : directeur de cabinet du préfet, préfet délégué et préfet, officier de police judiciaire, procureur, juge d'instruction. Comme le rappelle le code de déontologie de la police nationale (articles 13 à 18), «l'autorité de commandement prend les décisions... les traduit par des ordres... que le fonctionnaire de police doit exécuter loyalement». On est ainsi dans le modèle pur de l'autorité bureaucratique, légitimée par la position relative dans une hiérarchie de commandement continue, et procédant par instructions impératives, dont le modèle idéal est celui de l'armée (hors situation de combat). Toutes les observations externes du travail policier en milieu urbain, depuis un demi-siècle (Westley, 1950) et dans les environnements les plus divers, ont conclu que ce modèle était pure fiction, pour la raison structurelle déterminante qui tient au contrôle, par les exécutants sur le terrain, de l'information pertinente sur la nature de l'événement

Les commissaires de police qui constituent le corps de direction sont issus ainsi (en avril 2003) pour $62,5 \%$ du concours externe, qui suppose la détention d'un diplôme de l'enseignement supérieur, et pour le reste de la promotion interne (par concours, $25 \%$ ou «au choix», 10,9\%). Source: F. Ocqueteau (2004).

16. Consulter C. Dubar et P. Tripier (1998) et dans une littérature états-unienne immense, le récent E. Freidson (2001). 
sur lequel ils interviennent. Contrôlant ce paramètre essentiel de l'activité policière, ils disposent de fait d'une autonomie de décision considérable à l'égard de l'organisation hiérarchique tant pour le choix des tâches prioritaires que pour leur mode opératoire. L'explicitation de cette propriété mine radicalement les présupposés de l'organisation hiérarchique selon lesquels les subordonnés se contenteraient d'appliquer les consignes qui leur sont données.

Pendant longtemps, ce mode de fonctionnement a pu rester entièrement informel sans mettre en péril l'organisation, et d'abord parce que nul n'avait intérêt à le mettre en évidence. La fiction de l'autorité bureaucratique sert évidemment ceux qui occupent les postes d'autorité, mais elle permet aussi aux échelons subordonnés de ne pas assumer en droit les responsabilités de l'autonomie qu'ils exercent en fait. Tout le monde y trouvait ainsi son compte. Ce compromis n'était périodiquement menacé que localement, par les inspecteurs excipant de leur qualification personnelle d'officier de police judiciaire $\left.(\mathrm{OP})^{17}\right)$, pour s'émanciper de l'autorité hiérarchique. Situation particulière sans doute, mais dont il faut noter qu'elle est au principe de la très vive hostilité dont se marquent traditionnellement les organisations professionnelles des inspecteurs et des commissaires. Or cette situation, qui restait confinée dans cette zone particulière de l'espace policier, est en voie de généralisation. Trois facteurs y poussent.

En premier lieu, l'accroissement continu du niveau de recrutement des différents corps, et notamment du premier (gardiens de la paix), dont la majorité des recrues détient maintenant au moins le baccalauréat, nourrit un double mouvement d'élargissement des tâches de la part des exécutants et de reflux des formes les plus frustres du commandement hiérarchique. L'accession nouvelle de gardiens de la paix à la qualification OPJ est un bon indicateur de ce mouvement, qui permet le cas échéant à un gardien de détenir des compétences, et des pouvoirs, dont son encadrement direct est dépourvu.

Dans le même temps, l'évolution de la demande sociale de police, telle qu'elle se traduit par la mise en œuvre de nouvelles stratégies policières

17. La qualification d'OPJ est un attribut individuel, décerné et contrôlé par le parquet, et qui «qualifie» le policier qui la détient à prendre des mesures coercitives (garde à vue, par exemple), à la demande et sous le contrôle du magistrat. Dans l'exercice de cette qualification, son détenteur est indépendant de l'autorité hiérarchique. Il s'agit de fait d'un cas pur de disjonction entre compétence professionnelle et organisation hiérarchique. 
(police de proximité, police communautaire, police de résolution de problèmes (Brodeur, 2003)), implique aussi élargissement des tâches, et surtout multiplication des partenariats finalisés dans lesquels sont engagés les policiers. Ceux-ci sont, par le fait même, amenés à diversifier considérablement leurs compétences et surtout les instances auprès desquelles ils sont redevables, et sous ces deux aspects à mobiliser les ressources personnelles opposables à l'organisation hiérarchique.

Enfin, la montée régulière des statistiques de la délinquance, du sentiment d'insécurité et de sa politisation, a incité l'autorité politique s'exerçant sur la police à exiger de celle-ci, de façon de plus en plus pressante, des «résultats». La mise en ouvre de compstat dans la police new-yorkaise, et son extension très rapide dans les villes états-uniennes, illustre les effets de cette pression nouvelle sur les appareils policiers. On sait ainsi qu'une des premières mesures accompagnant l'invention des procédures «compstat» par l'équipe de $\mathrm{W}$. Bratton a été le limogeage, en l'espace d'une année, des deux tiers des 67 chefs de "precincts» (commissariats de quartier) de la ville (Silverman, 2001), pour incompétence, insuffisance, ou tentative de truquer les comptes. Si l'exemple reste isolé dans sa brutalité, il pointe une question plus générale: dans quelle mesure la hiérarchie existante est-elle légitimée par ses compétences opérationnelles? On ose ici soupçonner que la réponse donnée à New York serait transposable dans d'autres sites, il n'en reste pas moins qu'elle a pointé la disjonction possible entre position hiérarchique et compétence professionnelle, lorsque la compétence professionnelle doit se mesurer par rapport à la compétence opérationnelle.

Cette identification ne va pas de soi. Dans une grande organisation bureaucratique, la compétence qui légitime la position hiérarchique peut avoir de multiples fondements distincts, concurrents, alternatifs ou cumulables. Elle peut être fondée sur les performances scolaires, qui conditionnent le rang de sortie de l'école de police ${ }^{18}$, sur les compétences relationnelles, qui mettent en position d'être remarqué, sur la maitrise des instruments de gestion qui satisfait les bureaux, sur l'anticipation des priorités de l'échelon supérieur, sur la satisfaction au travail des subordonnés, ou sur les performances opérationnelles, qui requièrent elles-mêmes des habiletés précises (capacités de mobilisation des personnels, par exemple). (2004).

18. Et, par là, prédétermine largement la carrière future, voir sur ce point F. Ocqueteau 
L'objectivation des savoirs, qualifications et savoir-faire policiers conduit inévitablement à l'explicitation de ces différentes acceptions de la «compétence», et permet d'identifier leur répartition et hiérarchisation pratique dans l'organisation. L'administration policière en France témoigne d'une vulnérabilité particulière devant cet exercice. Dans une police de type anglo-saxonne, le recrutement initial unique permet de supposer que les carrières différentielles traduisent la hiérarchie des compétences. Autrement dit, le système repose sur le postulat que ce sont les meilleurs policiers qui sont promus, et que donc les postes les plus élevés sont détenus par ceux qui détiennent les compétences professionnelles les plus élevées. L'expérience de compstat et le limogeage massif qui l'accompagna, témoignent que ce n'est pas toujours et partout le cas, mais aussi qu'il existe des épreuves proprement professionnelles qui permettent, le cas échéant, de rétablir la légitimité de la hiérarchie. Dans le système français, la pluralité des voies d'entrée place automatiquement en position hiérarchique supérieure des recrues qui n'ont pas eu à témoigner d'une compétence spécialisée; on suppose qu'ils l'acquerront, il n'est pratiquement pas d'exemple que le défaut en soit sanctionné. Cette situation a pu se maintenir en longue période sous le double effet de l'opacité soigneusement entretenue de l'organisation policière et de son isolationnisme. Lorsque les évolutions sociales exogènes mettent la police «à la question», c'est bien le poids relatif en son sein de l'organisation bureaucratique et de la compétence professionnelle (opérationnelle) qui est aussi questionné. Et la hiérarchie en place, qui tient de cette place même le principe de sa légitimité, n'est pas spontanément encline à expliciter les contenus de la compétence supposée associée à ces places.

L'enjeu de la connaissance, et de l'objectivation, des savoirs policiers apparaît ainsi décisif. Le péril n'est pas tant externe, dans le caractère supposé subversif du regard des sciences sociales, mais interne: dans l'interrogation des ressources de légitimation de l'autorité dans la police. Celles-ci peuvent être bureaucratiques tant qu'on n'attend de la police que de la docilité et de la discipline. Si on lui fixe des objectifs, requiert des résultats, demande des comptes, ce sont les qualifications opérationnelles qui vont devenir premières, et donc la détention de savoirs proprement professionnels. Il ne s'agit pas là seulement d'un changement dans la logique de fonctionnement, il emporte nécessairement une évolution des rapports de force internes, des distributions de sanctions 
(positives et négatives), bref un bouleversement des positions et routines établies. De ce péril, la recherche est une métaphore. En autant qu'elle ait vocation à objectiver (ou mettre en relation) le fonctionnement policier, elle donne potentiellement à ceux qui entendent le changer les outils cognitifs requis. Elle donne à l'organisation la capacité d'être moins opaque à elle-même, et donc d'expliciter les enjeux d'une professionnalisation réelle, c'est-à-dire construite sur des savoirs. Il y a en somme réciprocité de perspective entre le savoir que les sciences sociales tentent de constituer sur la police, et au sein de celle-ci la place qui est donnée aux détenteurs du savoir professionnel, par rapport aux détenteurs des ressources bureaucratiques. On comprend alors assez rationnellement pourquoi ces derniers expriment à l'égard de la recherche une hostilité véhémente, et pourquoi il arrive parfois que la même recherche trouve une écoute parmi ceux qui prennent au sérieux le projet de professionnalisation de leur institution (Molin, 2001; Monet, 1985).

\section{Conclusion}

Les chercheurs peuvent être médiocres, et les recherches de qualité ou de pertinence inégales, mais l'attitude à l'égard de la recherche ellemême porte une autre signification et exprime des choix implicites. Au regard de leurs homologues dans les pays comparables, qui voient se multiplier les structures de recherche sur la police ${ }^{19}$, il semble bien que l'attitude dominante au sein de la hiérarchie policière française, de l'hostilité explicite à l'indifférence absolue, témoigne d'options majeures au regard du métier : le privilège accordé au rang et à la place plutôt qu'à la compétence et au savoir, la défense de la corporation plus que la

19. Parmi nombre d'indicateurs, voir la création d'un département de recherche à l'école de police de Catalogne (Revista Catalana de Seguretat Pública, n ${ }^{\circ} 8,2001$ ) et à l'École nationale de police du Québec (Les objectifs de recherche en matière policière, actes du colloque des 27 et 28 novembre 2001, ENPQ, Nicolet, 2002), la création en Belgique, sous l'égide conjointe des universités, écoles de police et administrations policières d'un "Centre d'études sur la police» (Étudier la police: une nécessité démocratique, CEP, Jurbise, 2004). Dans le récent bilan des recherches sur la police aux États-Unis, les responsables du groupe de travail de la National Academy (Skogan et Frydl, 2004), notent la "growing openness» des polices à l'égard de la recherche. On me permettra d'y ajouter le témoignage de l'accueil exceptionnellement ouvert que m'a consenti depuis 1998 le service de police de la ville de Montréal (SPVM), dont les dirigeants - au premier rang desquels M. Sarrazin - m'ont donné toute liberté, et toute la documentation requise, pour étudier sur place la réforme de la police de quartier (ouvrage en préparation). 
promotion de la profession. La tension inhérente à la position de «professionnels dans l'organisation» a été ainsi esquivée en donnant en tout et sur tout priorité aux valeurs de l'organisation, et en donnant aux «locaux» le pas sur les «cosmopolites» (Gouldner, 1957). Il n'est pas incohérent de ce fait que ce groupe, qui a su se doter d'une représentation syndicale puissante, active et quasi hégémonique, n'ait jamais été tenté de créer une association professionnelle ${ }^{20}$.

Il n'est pas assuré que cette situation soit durable. Les facteurs de tension que nous avons relevés: accroissement du bagage scolaire des recrues, découplement des grades et de la qualification (OPJ), expériences du partenariat, pressions de la demande sociale et instillation d'une culture du résultat vont vraisemblablement continuer à agir. Il deviendra alors de plus en plus périlleux de fonder la légitimité des normes traditionnelles de fonctionnement de l'organisation, et de sa hiérarchie, sur les seuls arguments d'autorité, gagés sur l'insularité et l'opacité. Il faudra attester de compétences et de savoirs. On peut supposer que cette tension s'incarnera dans des fractions distinctes du corps policier, et que les segments les plus professionnels en viendront à considérer, comme on l'observe dans nombre de polices occidentales, la recherche en sciences sociales comme grosse de ressources ${ }^{21}$, et non plus de menaces. On a parfois voulu croire, dans les cercles policiers les plus conservateurs, que les succès de la police new-yorkaise étaient fondés sur une idéologie de «tolérance zéro». Tout ce que la recherche nous en apprend démontre beaucoup plus sûrement que la popularité du modèle new-yorkais

20. «De source généralement bien informée», on ajoute que le corps de direction de la police nationale est aussi très bien représenté dans les organisations maçonniques. L'accueil réservé par le $31^{\mathrm{e}}$ congrès du Syndicat des Commissaires (SCHFPN) au grand maître du Grand Orient, principale loge française, ne dément pas cette information, qui soutient le constat d'un privilège accordé aux ressources relationnelles sur les ressources professionnelles. Voir La tribune du commissaire de police (2001, vol. 82: $52-55$ et 83:32-39). Dans ce même numéro (2001, vol. 83 : 38), l' IHÉSI est qualifié d'«Institut des hautes études sans intérêt». Nul doute que les auteurs de cette formule et $A$. Bauer qui y souscrit aient pris, pour nourrir ce jugement, connaissance attentive des recherches commanditées par l'Institut dont on trouvera la liste en annexe ci-après.

21. Comme l'ont été, pour la fraction modernisatrice des chefs de police nord-américains, les travaux de la Police Foundation (Washington) dans les années 60 et 70 . Pour une illustration plus récente, on peut aussi consulter les programmes de recherche développés depuis deux lustres sous l'égide du National Institute of Justice (ministère fédéral de la Justice) dans le champ du «community policing». Dans les deux cas, la coopération entre services de police territoriaux, agences publiques et communauté scientifique apparât (à l'observateur français tout au moins) exemplaire. 
résulte d'abord de la décision initiale de ses responsables «d'ouvrir toutes grandes les portes du département à la presse, aux chercheurs et à tous autres »(Silverman, 2001). Tant il est vrai que le professionnalisme, lorsqu'il est assuré de lui-même, ne requiert pas le secret.

\section{Références}

Auteur inconnu. (2001) La tribune du commissaire de police, 82, 52-55.

Auteur inconnu. (2001) La tribune du commissaire de police, 83, 32-39.

Bauer, A. (2001). Intervention. La tribune du commissaire de police, 82, 52-55.

Brodeur, J.-B. (2003). Les visages de la police. Montréal: Les Presses de l'Université de Montréal.

Brodeur, J.-P. (1984) La police, mythe et réalité. Criminologie, XVII, (1), 9-41.

Chauvenet, A. \& Orlic, F. (1985). Interroger la police. Sociologie du travail, XXVII, (4), 453-467.

CNRS (1984). La police quotidienne, éléments de sociologie du travail policier. Dans: Groupe de sociologie du travail.

Collection. (1993). Police et médias. Cabiers de la sécurité intérieure, 12.

Dubar, C. \& Tripier, P. (1998). Sociologie des professions. Paris: Armand Colin.

École nationale de police du Québec. (2002). Les objectifs de recherche en matière policière, actes du colloque des 27 et 28 novembre 2001, Nicolet: ENPQ.

Fielding, N. (1988). Joining forces, police training, socialization and occupational competence. Londres: Routledge.

Freidson, E. (2001). Professionalism, the third logic. Cambridge: Polity Press.

Gouldner, A. W. (1957). Cosmopolitans and locals, toward an analysis of latent social roles. Administrative Science Quarterly, 2 (3), 281-306.

Kornhauser, W. (1962). Scientists in industry, conflict and accommodation. Berkeley: UC Press.

Latour, B. (2002). La fabrique du droit, une ethnographie du conseil d'État. Paris: La Découverte

Loubet del Bayle, J. L. (1999). Jalons pour une histoire de la recherche française sur les institutions et les pratiques policières. Les Cabiers de la Sécurité Intérieure, 37, 55-71.

Ministère de l'Intérieur (1982). Les policiers, leur métier, leur formation. Paris: La documentation française.

Ministère de l'Intérieur. (2003). Rapport annuel. Paris.

Molin, F. (2001). Les sciences humaines et la formation dans la police nationale, paradoxes, malentendus et perspectives. Les Cabiers de la sécurité intérieure, 46, 47-58.

Monet, J.-C. (1985). Une administration face à son avenir: police et sciences sociales. Sociologie du travail, XXVII, (4), 370-390. 
Monjardet, D. (1988). Le maintien de l'ordre, technique et idéologie professionnelle des C.R.S., Déviance et Société, 12 (2), 101-126

Monjardet, D. (1996). Ce que fait la police. Paris: La Découverte.

Monjardet, D. (2002). L'insécurité politique, police et sécurité dans l'arène électorale. Sociologie du travail, 44, (4), 543-555.

Monjardet, D. (avril 1997). Le chercheur et le policier: l'expérience des recherches commanditées par le ministère de l'Intérieur. Revue française de science politique, 47, (2), 29-42

Monjardet, D. et Gorgeon, C. (1993). La socialisation professionnelle des gardiens de la paix. Paris: INHES.

Ocqueteau, F. (2004). Les commissaires de police en quête d'identité professionnelle. Rapport CERSA, Paris: INHES.

Ocqueteau, F. \& Monjardet, D. (2005). Indispensable et insupportable, la recherche au ministère de l'Intérieur. In Ph. Bezes, M. Chavière, J. Chevallier, N. De Monbicher \& F. Ocqueteau, L'État à l'épreuve des sciences sociales (229247), Paris: La découverte.

Police de la ville de Montréal (SPVM) (1998). Document interne.

Pomes I. \& Abella, X. (2001). Presentacion. Revista Catalana de Seguretat Publica, 7-11.

Punch, M. (1986). The politics and etbics of field work. Beverly Hills: Sage.

Reiner, R. (1993). Du mythe à la réalité : le modèle britannique. Les Cabiers de la sécurité intérieure, 13, 17-59.

Rudolph, L. (2001). Le policier et le chercheur. Les Cabiers de la sécurité intérieure, $46,19-30$.

Sans auteur. (2001). Questions à M. Alain Bauer. La tribune du commissaire de police. $83,32-38$.

Silverman, E. B. (2001). NYPD battles crime, innovative strategies in policing $\left(2^{\text {nd }}\right.$ edition). Boston: Northwestern U.P.

Skogan, V. \& Frydl, K. (dir.) (2004). National Research Council, Fairness and effectiveness in policing: the evidence. Washington, DC: The National Academy Press.

Skolnick, J. (1966). Justice without trial. NY: Wiley.

Tiévant, S. (2001). Les savoir-faire en police de proximité. Paris: IHÉSI.

Vav Maanen, J. (1975). Police socialization. Administrative Science Quarterly, 20 (2), 207-228.

Westley, W. (1970). Violence and the police. Cambridge: MIT Press 


\section{A N NEXE \\ Rapports de recherches commanditées par l'IHÉSI de 1990 à 2004, ayant pour objet police ou gendarmerie en France.}

1991

BERGES, Michel

BERLIÈRE, Jean-Marc

VOGEL, Marie

TIEVANT, Sophie

WIEVIORKA, Michel

MOSER, Gabriel

PASCUAL, Angélina

DELCROIX, Catherine

CUNHA, Maria

MOUHANNA, Christian
La pluralité des modèles policiers français: inventaire historique

La mission de sécurisation: étude du dispositif des patrouilles CRS en renfort de la PU

Sociologie du racisme: police et racisme

L'image de la police à Marseille: les quartiers «Nord» et le «centre-ville»

Pertinence du territoire et de la fonction d'une police de proximité - Exploration d'un espace social: Montfermeil

Étude sur l'expérience d'îlotage à Roubaix

1992

JANKOWSKI, Barbara

DULONG, Renaud RELIEU, Marc

RENOUARD, Jean-Marie

PAPERMAN, Patricia

ROBERT, Jean-Philippe
Étude auprès des policiers auxiliaires

Analyse sociologique d'une intervention filmée de la police

Les relations entre la police et les jeunes: la recherche en question

Vision en sous-sol: la vie quotidienne des policiers dans le métro

L'image de la police dans l'agglomération de Lille, Roubaix, Tourcoing

GORGEON, Catherine et al.Les brigades de roulement en police urbaine: fonctionnement, contraintes et potentialités

GATTO, Dominique La sécurité publique à l'épreuve du terrain. Le policier,

THOENIG, Jean-Claude le magistrat et le préfet, IHÉSI / L'HARMATTAN, 1992

MONJARDET, Dominique GORGEON, Catherine

1167 recrues. Description de la $121^{\text {e }}$ promotion des élèvesgardiens de la paix de la police nationale

JANKOWSKI, Barbara

GORGEON, Catherine

La police de proximité: regard sur un nouveau style de police

Police et public: représentations, recours, attentes

\section{3}

DOURLENS, Christine

VIDAL-NAQUET, Pierre

L'autorité comme prestation. La justice et la police dans la politique de la ville

MONJARDET, Dominique GORGEON, Catherine
La socialisation professionnelle des policiers.

Étude longitudinale de la $121^{\mathrm{e}}$ promotion des élèves gardiens de la paix. La formation initiale. Tome I 
THOENIG J., Claude BERGES, Michel

BOURGEOIS, Frédérique

DIEPPEDALE, Dominique

DUPREZ, Dominique

LEMAITRE, Rémi

LAZERGES, Christine
L'étatisation des polices municipales.

Bordeaux et le pouvoir central: 1884, 1941

La police à l'épreuve du social

La gestion de l'immigration irrégulière. Analyse sociologique des identités professionnelles des policiers et des douaniers

Les relations police/justice dans le cadre de la garde à vue. L'exemple de Lyon

\section{4}

BUGNON, Françoise

DARTEVELLE, Michel

JANKOWSKI, Barbara

TIEVANT, Sophie

MOUHANNA, Christian ACKERMANN, Werner
La socialisation des jeunes gardiens de la paix

De l'appel au 17 à l'intervention d'un équipage: perspectives de recherche et études du travail en salle de trafic et de commandement à Lyon

Attachement au métier et isolement institutionnel: les inspecteurs de sécurité publique. Enquête dans cinq commissariats de la région parisienne

L'activité de la gendarmerie et la sécurité des habitants dans les zones urbaines

Une affaire de confiance. Les relations OPJ-magistrats dans le processus pénal

\section{7}

BOULLIER, Dominique CHEVRIER, Stéphane MIDOL, André

MEISSONNIER, Joël

MONJARDET, Dominique GORGEON, Catherine

VOGEL Marie

HEILMANN, Éric GAUTHIER, Yves

MEADEL, Cécile AKRICH, Madeleine
L'activité des forces de police et de gendarmerie en matière de sécurité routière

La collaboration des métiers de l'urgence (polices/sapeurspompiers) avec les travailleurs sociaux. Les affaires de violences domestiques

La socialisation professionnelle des policiers.

Étude longitudinale de la $121^{\mathrm{e}}$ promotion des élèves gardiens de la paix. Tome II : La titularisation

Bibliographie sur la police

Europol et la coopération policière en Europe: actions et perspectives ouvertes par le traité sur l'union européenne

Polices et technologies informatiques. Nouveaux traitements de l'information et gestion de l'activité

\section{7}

BOULLIER, Dominique LEGRAND, Marc

CHAMBRON, Nicole IMPERIALI, Françoise
La rareté du témoignage. Le travail de production du témoin par la police judiciaire

Les relations entre policiers et travailleurs sociaux: les conditions d'une coopération 
BERLIÈRE Jean-Marc PESCHANSKY, Denis

MIDOL, André

MÉNARD, Pascale

MOUHANNA, Christian

DIEU, François

CETTINA, Nathalie

BUGNON, Françoise
Pouvoirs et police au XXe siècle

La gestion des plaintes et réponse à l'insécurité

Quelles évolutions pour la police de proximité? L'exemple de Montfermeil 1991-1996

Sécurité et ruralité. Enquête sur l'action de la gendarmerie dans les campagnes françaises

La coordination policière et judiciaire dans la lutte contre le terrorisme en France

Relations entre travail et hors-travail chez des policiers de province: la carrière, la famille, la maison et la participation à la vie locale et associative

1998

DIEU, François Les charges de travail des gendarmes

1999

MIGNON, Paul

L'activité de la police nationale et de la gendarmerie nationale face à la demande sociale de sûreté publique dans de petites villes

MOUHANNA, Christian Une police de proximité judiciarisée: de l'îlotage au renseignement judiciaire

MONJARDET, Dominique La socialisation professionnelle des policiers.

GORGEON, Catherine Étude longitudinale de la $121^{\mathrm{e}}$ promotion des élèves-gardiens de la paix. La banalisation. Tome III

2000

MOUHANNA Christian Quel service pour quel public? Une tentative d'évaluation chiffrée de l'image de la police dans la population face à la territorialisation

2001

TIEVANT, Sophie

Caractérisation des savoir-faire spécifiques en police de proximité et examen des moyens de leur acquisition

DUPREZ, Dominique PINET, Michel

Policiers et médiateurs, sur le recrutement et les appartenances

WUILLEUMIER, Anne

Création et développement d'un service de la police nationale: le cas des brigades régionales d'enquêtes et de coordination (BREC) de la police judiciaire

2002

KATANE, David

La police et la ville. Questions sur la proximité

ROCHÉ, Sebastian

Les réactions à la délinquance des jeunes: la police, la justice ASTOR, Sandrine 
2003

ESNARD, Catherine

Changements organisationnels et représentations des récentes

LECIGNE, André réformes chez les personnels de la police nationale

2004

DIEU, François

La gendarmerie et la police de la route

MONJARDET, Dominique La socialisation professionnelle des policiers, dix ans plus tard. GORGEON, Catherine «La cristallisation»

MIDOL, André

Police de proximité et circulation de l'information entre services de police

OCQUETEAU, Frédéric Les commissaires de police en quête d'identité professionnelle

MOREAU de BELLAING, Policer les policiers. Le travail de l'Inspection générale des Cédric services

PECAUD, Dominique Culture, normes et régulation professionnelles dans la police

PRUVOST, Geneviève 1982-2003: enquête sociodémographique sur les conditions COULANGEON, Philippe de vie et d'emploi de 5221 policiers

ROHARIK, Ionela

On compte ainsi 59 recherches ayant pour objet, au moins partiel, police ou gendarmerie en France, soit $32 \%$ de l'ensemble des recherches engagées par l'IHÉSI durant la période $(n=185)$. 